\title{
Evaluación del exponente de la función de corrección de la velocidad de sedimentación de Richardson y Zaki para micro partículas
}

Recepción: Febrero de 2007 / Aceptación: Mayo de 2007

(1) Gonzalo Salinas Salas (2) Frank Babick

\begin{abstract}
RESUMEN
La función de corrección formulada por Richardson y Zaki en 1954, se utiliza para determinar la velocidad de sedimentación de un colectivo de partículas a partir de la velocidad de una partícula que no se encuentra sujeta a la acción de las fuerzas de otras partículas en la suspensión. Dado el tamaño de las partículas que es micrométrico y el valor del número de Reynolds del escurriento que es menor a 0,25 ; se emplea la solución de Stokes para evaluar la velocidad de éstas, por lo que el valor del exponente de la función de corrección de Richardson y Zaki es $n=4,65$; independientemente del tamaño. El presente trabajo demuestra a partir de un trabajo experimental con micro partículas que el valor del exponente de la función de corrección de Richardson y Zaki depende de su tamaño y es mayor mientras menor es el diametro de éstas.
\end{abstract}

Palabras clave: Función de corrección, velocidad de sedimentación, micropartículas, trabajo experimental, exponente.

EVALUATION OF THE EXPONENT OF THE R I C H A R D S O N A N D Z A K I SED I M EN T A T I ON VEL OCIT Y CORRECTION FUNCTION FOR MICRO PARTICLES

ABSTRACT

Richardson and Zaki (1954) formulated a correction function for determining the sedimentation velocity of a suspension of particles on the basis of the velocity of a single particle not subject to forces other particles in the suspension. The Reynolds number for the flow is less than 0.25 , so the Stokes equation can be used to calculate their velocity. The exponent in the Richardson and Zaki correction function is $n=4.65$, independent of size. This paper describes research with micro particles in which the value of the exponent of the Richardson and Zaki correction function was found to be inversely dependent on the size of the micro particles.

Keywords: Correction function, sedimentation velocity, micro particles, experimental research, exponent

\section{INTRODUCCIÓN}

La función de corrección propuesta por Richardson y Zaki [2], se emplea para corregir la evaluación de la velocidad de sedimentación de un conjunto de partículas en un fluido viscoso, a partir del calculo de la velocidad de sumergimiento terminal de una única partícula de igual diámetro medio en el mismo fluido, a fin de considerar en esta evaluación los efectos de las fuerzas hidrodinámicas y electroquímicas que se presentan por la interacción que tienen entre sí las distintas partículas que se sumergen simultáneamente.

Respecto de las fuerzas hidrodinámicas se puede indicar que se originan a partir de la formación de puentes hidráulicos entre partículas y/o por el arrastre que las partículas de mayor diámetro realizan sobre las de un menor diámetro, lo que altera tanto la velocidad de sumergimiento como la trayectoria de ésta. A lo anterior se suma el efecto dominante que tienen las fuerzas de superficie tipo electroquímico entre las partículas, las que pueden definirse a través del potencial Zeta de las partículas. Estas fuerzas son relevantes en el caso de micropartículas, puesto que las superficies expuestas al electrolito, que en este caso es el fluido, son muy significativas por la gran superficie expuesta. Estas fuerzas que se les denominan como fuerzas débiles son modeladas a través de la teoría DLVO, desarrollada por Derjarin, Landau, Verwey y Overbeck [3].

La suma de estos efectos genera una disminución de la velocidad de sedimentación de un colectivo de partículas, la cual ha sido modelada a partir de consideraciones hidrodinámicas y empíricas por diversos autores. Sin embargo, la modelación semi empírica de Richardson y Zaki es la más comúnmente utilizada actualmente, para el diseño de sistemas de sedimentación como de sistemas de medición de distribución de tamaño partículas, sistemas de tratamiento de residuos y otras aplicaciones industriales [1], [3] y [4].

Matemáticamente la función de corrección de Richardson y Zaki se plantea a partir de la denominada velocidad terminal de sedimentación, que es la velocidad con la cual una partícula alcanza un equilibrio dinámico en el fluido entre su peso propio, la fuerza de flotación y la fuerza de arrastre viscoso, alcanzando una velocidad de sumergimiento constante y por ello es posible emplear la expresión de la fuerza de arrastre viscoso, a partir del coeficiente de arrastre definido por las ecuaciones de Stokes o de Martín o de Newton, según el número de Reynolds que alcance el escurrimiento del fluido sobre la micro particula [4].

\footnotetext{
(1) Doctor en Ingeniería (c). Profesor del Departamento de Tecnologías Industriales. Universidad de Talca. Chile E-mail:gsalinas@utalca.cl

(2) Doctor en Ingeniería. Profesor de la Technische Universität Dresden. Alemania

E-mail:mechanvt@rcs.urz.tu-dresden.de
} 


\begin{tabular}{|c|c|}
\hline \multicolumn{2}{|c|}{ Cuadro 1. Exponentes de la función de Richardson y Zaki [3] } \\
\hline Rango de número de Reynolds & Exponente $\mathbf{n}$ \\
\hline $\operatorname{Re} \leq 0,2$ & 4,65 \\
\hline $0,2<\operatorname{Re}<1$ & $4,35 \cdot \operatorname{Re}^{0,03}$ \\
\hline $1<\operatorname{Re}<500$ & $4,45 \cdot \operatorname{Re}^{0,1}$ \\
\hline $500<\operatorname{Re}<300000$ & 2,39 \\
\hline
\end{tabular}

La forma general de la función de corrección de Richardson y Zaki es presenta mediante la ecuación (1).

$$
\mathrm{FC}_{\mathrm{R} \cdot \mathrm{z}} \cdot \frac{\mathrm{v}_{\text {sumergimiento }}}{\mathrm{v}_{\text {Stokes }}} \cdot \cdot 1 \cdot \mathrm{c} \cdot{ }^{\mathrm{n}}
$$

Los trabajos de Richardson y Zaki, demostraron que existía una dependencia entre el valor del número de Reynolds (Re), alcanzado por el escurrimiento y el valor del exponente de la función de corrección (n), lo que se tradujo en el Cuadro 1.

A su vez, la velocidad de Stokes se evalúa de la siguiente manera:

$v_{\text {Stokes }} \cdot \frac{\left(\rho_{p} \cdot \rho_{f}\right) \cdot d^{2} \cdot z \cdot g}{18 \cdot \mu}$

Por su lado, el factor de aceleración, z, se determina para el caso de campo centrifugo como:

$z \cdot \frac{\omega^{2} \cdot r}{g}$

Finalmente, el número de Reynolds para el caso de partículas esféricas está definido como:

$\operatorname{Re} \cdot \frac{d \cdot \rho_{f} \cdot v_{\text {sumergimiento }}}{\mu}$

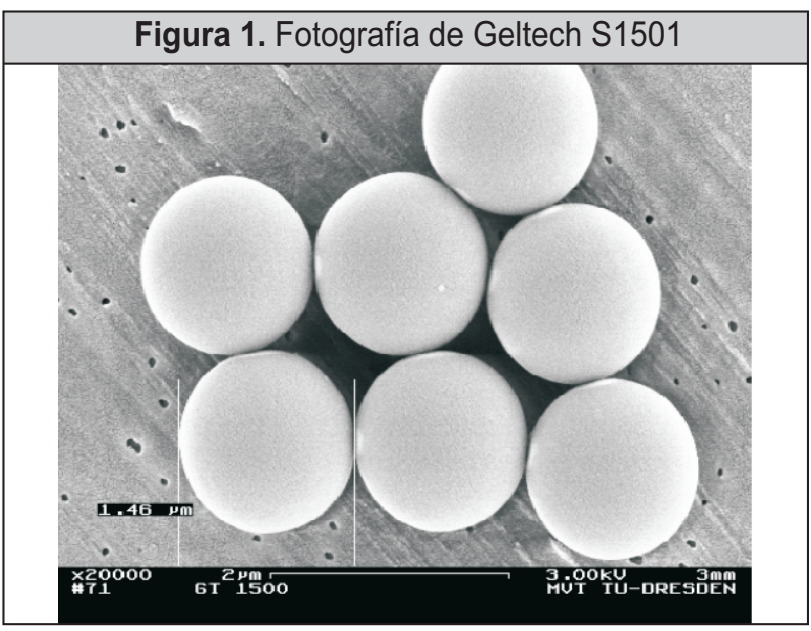

\begin{tabular}{|c|c|c|}
\hline \multicolumn{3}{|c|}{ Cuadro 2. Datos técnicos del material } \\
\hline Diámetro nominal & Código del material & Fracción $\mathbf{5 0 \%}$ \\
\hline $1,5 \mu \mathrm{m}$ & $\mathrm{S} 1501$ & $1,39 \mu \mathrm{m}$ \\
\hline $1,0 \mu \mathrm{m}$ & $\mathrm{S} 1001$ & $1,06 \mu \mathrm{m}$ \\
\hline
\end{tabular}

Como se aprecia el valor del exponente de la función de corrección de Richardson y Zaki empleado para el caso de las micro partículas que sedimentan en regímenes de escurrimiento con números de Reynolds menores a 0,25 es 4,65, independientemente de su tamaño.

El presente trabajo muestra que la dependencia entre el valor del exponente de la función de corrección de Richardson y Zaki, se mantiene a medida que el tamaño de las partículas que sedimentan disminuye, como el valor de número de Reynolds del escurrimiento, lo que se traduce en valores mayores del exponente de la función de corrección de Richardson y Zaki. Este comportamiento se determinó a partir de un trabajo de carácter netamente experimental desarrollado con micropartículas calibradas de óxido de silicio $\left(\mathrm{SiO}_{2}\right)$ suspendidas en agua con hidróxido de potasio $(\mathrm{KOH})$ como agente dispersante, siguiendo la norma de ensayo de sedimentación DIN 66111, que es válida actualmente para este tipo de ensayos [1].

\section{TRABAJO EXPERIMENTAL}

Las micro partículas utilizadas en este trabajo son estándares calibrados de tipo comercial, proveídas por la empresa Geltech Inc. Químicamente estas micro partículas son dióxido de silicio amorfo $\left(\mathrm{SiO}_{2}\right)$, de forma esférica y rígidas, con una densidad del

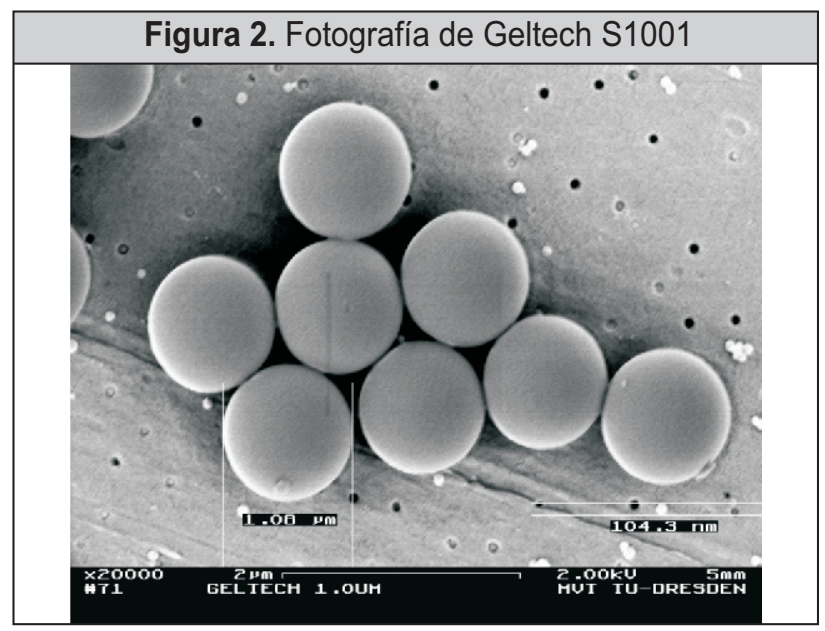




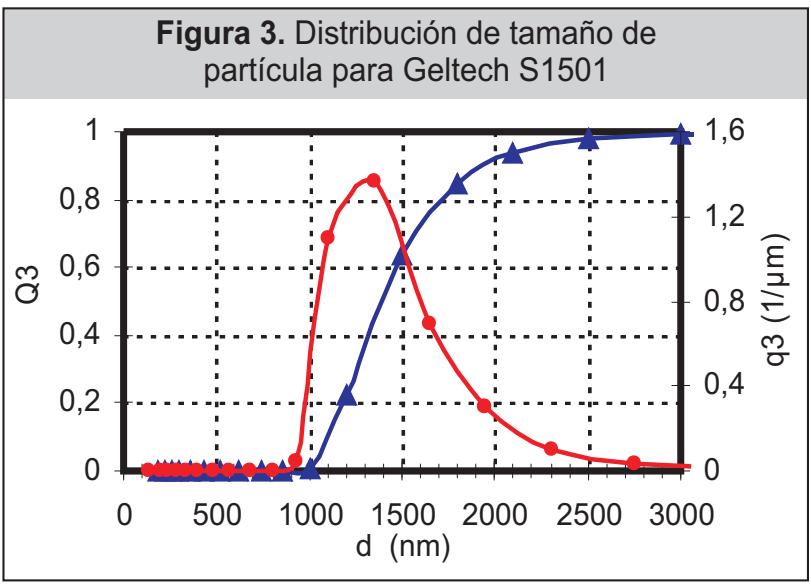

orden de $2.135 \mathrm{~kg} / \mathrm{m}^{3}$, con diámetros nominales de $1,5 \mu \mathrm{m}$ y $1,0 \mu \mathrm{m}$. El Cuadro 2 presenta las principales características del material utilizado.

Por su lado, las Figuras 1 y 2, muestran fotografías que fueron obtenidas durante los ensayos a través de un microscopio electrónico Zeiss Gemini 942, donde se muestra tanto la forma, como el tamaño de las micropartículas de Geltech utilizadas en los ensayos.

Respecto de las suspensiones, éstas se elaboraron con agua tridestilada, desionizada y filtrada, a la cual se le adicionó hidróxido de potasio $(\mathrm{KOH})$ a fin que alcanzara la suspensión un índice de acidez $(\mathrm{pH})$ del orden de 9,2, a fin de alcanzar un potencial Zeta, que dependiendo del tamaño de las partículas, fue del orden 40 a $60 \mathrm{mV}$, con el objeto de asegurar la estabilidad de la suspensión lo que permite inhibir la formación de flóculos.

La suspensión se sometió a una cuidadosa preparación siguiendo la Norma DIN 66111, a fin de evitar tanto la contaminación por micro partículas extrañas a las que conforman la suspensión de trabajo.

En cuanto al procedimiento de dispersión, este fue mediante agitación mecánica a alta velocidad y ultrasonido. Realizada la suspensión se procedió a verificar el estado de la suspensión mediante análisis de las imágenes aportadas por el microscopio electrónico Zeiss Gemini 942, que no revelaron la presencia de partículas extrañas a la suspensión de prueba y la determinación mediante espectrometría láser con un equipo Helos Fa. Sympatec de la distribución de tamaño de partícula, cuyos resultados se muestran en las figuras 3 y 4 , que indican que la dispersión del material particulado es adecuada y confirman la inexistencia de contaminación

En cuanto a la centrífuga analítica utilizada para desarrollar los ensayos ésta fue una máquina LUMiFuge 114, fabricada por L.U.M. GmbH, donde se

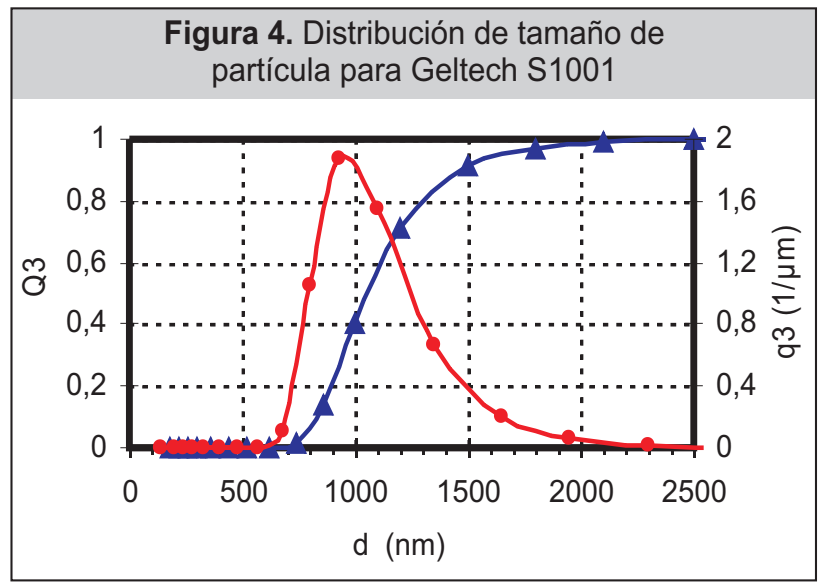

utilizaron cubetas del tipo rectangular de polystyrol de $3 \times 7 \mathrm{~mm}^{2}$. El análisis de imágenes y la determinación de la velocidad de sedimentación fue realizado mediante el software LUM View v. 2.0, provisto por la misma firma. Los principales datos técnicos del equipo se presentan en el Cuadro 3.

Como ya se mencionó la norma utilizada para el desarrollo de estos ensayos fue la DIN 66111 relativa a métodos de sedimentación y es aplicada desde 1998 [1]. Esta norma especifíca tanto los materiales a emplearse en un ensayo, esto es el fluido, las partículas y el agente dispersante, como los procedimientos mismos de, las condiciones ambientales y la forma de representar los resultados de las mediciones de distribución de tamaño de partículas alcanzados.

\section{RESULTADOS YDISCUSIÓN}

Los ensayos se realizaron en el Laboratorio de Partículas y Granumetría del Grupo de Trabajo de Técnicas de Procedimientos Mecánicos de la Universidad Técnica de Dresden (Arbeitgruppe für mechanische Verfahrenstechnik an der Technische Universität Dresden) y los resultados mostraron una tendencia a seguir la forma genérica de la función de corrección de Richardson y Zaki, pero con un valor del exponente de la función distinto al propuesto por estos [2], como lo muestra la figura 5, donde se contrastan los valores experimentales con los valores de la función de corrección que utiliza el exponente convencional ( $n \leq 4,65$ ), para escurrimientos con número de Reynolds menores a 0,25 , por lo que son aplicables a micro partículas menores a un diámetro medio de 1,5 $\mu \mathrm{m}$, sujetas a campos de aceleración centrífuga que dan lugar a regímenes de escurrimiento con números de Reynolds menores a 0,001 .

Este comportamiento es esperable, ya que a medida que el tamaño de las micro partículas que existen la 
Figura 5. Comparación entre valores experimentales y la función de Richardson y Zaki

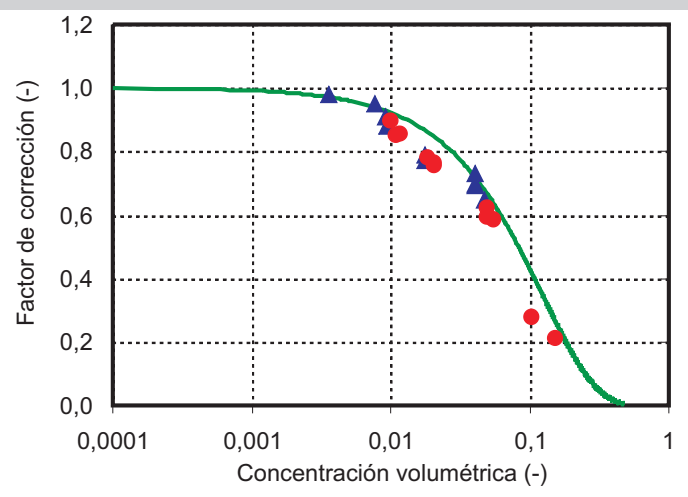

$\Delta$ Geltech $1500(\mathrm{~nm})$

- Geltech $1000(\mathrm{~nm})$

Richardson y Zaki; $n \leq 4,65$
Figura 6. Comparación entre valores experimentales y la función de Richardson y Zaki para distintos exponentes

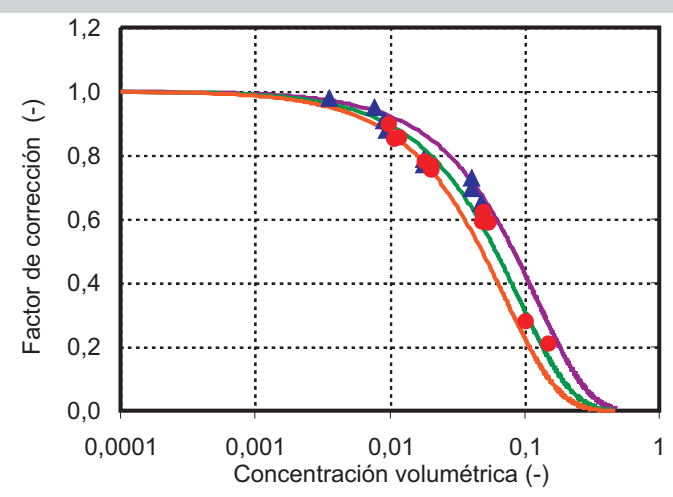

- Richadson y Zaki, $\mathrm{n} \leq 8$ - Richardson y Zaki, $\mathrm{n} \leq 11$

Richardson y Zaki, $\mathrm{n} \leq 14$ A Geltech $1500(\mathrm{~nm})$

Geltech $1000(\mathrm{~nm})$

Cuadro 3. Datos técnicos de la centrífuga analítica LUMiFuge 114

\begin{tabular}{|l|l|}
\hline Tipo de equipo & $\begin{array}{l}\text { Sistema de análisis de suspensiones } \\
\text { LUMiFuge }\end{array}$ \\
\hline Fabricante & L.U.M. GmbH \\
\hline Intervalo de medida & $10 \mathrm{~s}-600 \mathrm{~s}$ \\
\hline Velocidad de centrifug ación & $300-3.000 \mathrm{rpm}$ \\
\hline Fuente de luz & NIR-LED \\
\hline Longitud de onda en el espectro BW 50\% & $880 \mathrm{~nm}, 80 \mathrm{~nm}$ \\
\hline Foto sensor & CCD sensor de una fila \\
\hline Número de cubetas & 8 \\
\hline Cubetas & $\begin{array}{l}\text { Rectangulares de polystyrol, } 3 \times 7 \mathrm{~mm}^{2} \\
\text { Cilíndricas de vidrio, } \varnothing \leq 11,5 \mathrm{~mm}\end{array}$ \\
\hline Volumen de prueba & $0,5 \mathrm{~cm}^{3} ; 2 \mathrm{~cm}^{3} ; 0,15 \mathrm{~cm}^{3} ; 0,3 \mathrm{~cm}^{3}$ \\
\hline Rango de concentraciones volumétricas & $1 \%$ hasta $90 \%$ \\
\hline Rango de tamaños de partículas & $50 \mathrm{~nm}-100.000 \mathrm{~nm}$ \\
\hline Rango de densidad de partículas & $0,01 \mathrm{~g} / \mathrm{cm}^{3}-10 \mathrm{~g} / \mathrm{cm}^{3}$ \\
\hline Rango de velocidades de sedimentación & $1 \mathrm{~nm} / \mathrm{s}-750.000 \mathrm{~nm} / \mathrm{s}$ \\
\hline
\end{tabular}

Cuadro 4. Nomenclatura

\begin{tabular}{|l|l|}
\hline$F_{C_{R-Z}}$ & Factor de corrección de Richardson y Zaki \\
\hline$V_{\text {sumergimiento }}$ & Velocidad de sumergimiento del conjunto de partículas \\
\hline$V_{S t o k e s}$ & Velocidad de Stokes para la sedimentación de la partícula de tamaño medio \\
\hline $\mathrm{C}$ & Concentración volumétrica del colectivo de partículas \\
\hline $\mathrm{n}$ & Exponente de la función de corrección \\
\hline$\rho_{\mathrm{p}}$ & Densidad media de la partícula \\
\hline$\rho_{\mathrm{f}}$ & Densidad media del fluido \\
\hline $\mathrm{d}$ & Diámetro medio del conjunto de partículas \\
\hline $\mathrm{z}$ & Factor de aceleración \\
\hline$\omega$ & Velocidad rotacional \\
\hline $\mathrm{r}$ & Radio de rotación \\
\hline $\mathrm{g}$ & Aceleración de gravedad \\
\hline$\mu$ & Viscosidad dinámica del fluido \\
\hline $\operatorname{Re}$ & Número de Reynolds \\
\hline
\end{tabular}


suspensión disminuye, mayor es la cantidad de micro partículas presentes en ésta en un mismo valor de concentración volumétrica, esto conduce a varios efectos, siendo los dos principales:

a) Que tanto la masa como el volumen de las micro partículas de menor tamaño es obviamente menor al que tendrían micro partículas mayores y por ende las fuerzas mecánicas que actúan sobre las primeras son significativamente menores con respecto a las que se presentan en el caso de las segundas, esto conduce a que su magnitud relativa frente a las fuerzas de origen electroquímico para las micropartículas de menor diámetro se incrementen ya que la distancia entre las micro partículas disminuye, a la vez que la fuerza debida a la difusión osmótica aumenta, lo que se traduce en una redución con ello de la velocidad de sedimentación del conjunto de micropartículas.

b) El hecho de existir una mayor cantidad de micropartículas en la suspensión por efecto de su disminución de tamaño de éstas, conduce a que la magnitud del área de sus superficies en contacto con el fluido, que se comporta como un electrolito, aumente significativamente lo que implica que las fuerzas electroestáticas, de Van der Waals y de atracción de Born, representadas a través del potencial Zeta, aumenten siguiendo lo indicado por la teoría DLVO [3] y [4]. Esto implica un importante aumento de las fuerzas de repulsión entre micro partículas adyacentes y con ello la formación de una red de fuerzas entre las micro partículas que impide su sedimentación a velocidades similares a la de Stokes.

El hecho de emplear exponentes de mayor valor conduce a que el nuevo valor que toma el factor de corrección de Richardson y Zaki de cuenta de mejor manera estos fenómenos, mostrando que la reducción de la velocidad efectiva de sumergimiento de un conjunto de micro partículas respecto de la velocidad de Stokes evaluado para una micropartícula única, es mayor mientras menor sea el tamaño de las micropartículas que conforman la suspensión.

Como lo indica la figura 6 , donde se contrastaron los valores experimentales con la forma genérica de la función de Richardson y Zaki utilizando cifras mayores para el exponente, de manera que la función de corrección pudo así representar de una mejor manera la realidad del proceso de sumergimiento, demostrando de paso la directa relación que existe entre el valor que toma el exponente con el tamaño de las micropartículas que sedimentan.
Cabe indicar que al realizarse los experimentos de acuerdo a la norma DIN 66111, el utilizar un material de referencia calibrado y equipos de control como el microscopio electrónico y el espectrómetro láser, permite tener un buen nivel de confianza en los valores experimentales obtenidos, ya que éstos mostraron un buen nivel reproducibilidad, considerando la gran cantidad de ensayos y muestras empleadas, lo que se facilitó al emplear la centrífuga analítica LUMFuge 114, que permitía realizar ocho (8) ensayos simultáneos.

\section{CONCLUSIONES}

Se puede concluir que la evaluación de la velocidad de sedimentación de micro partículas realizados a partir de la velocidad de Stokes y que empleen la función de corrección de Richardson y Zaki con el exponente convencional ( $n \leq 4,65$ ), no representan adecuadamente el fenómeno, ya que muestran significativas discrepancias en su predicción de la velocidad de sumergimiento respecto de la que efectivamente se produce en un proceso que involucren suspensiones de partículas de tamaño micrométrico. Esto indica que los diseños de equipos de medida y de separación de fase y/o material particulado deban considerar esta situación a fin de incrementar tanto su precisión de medida como su eficiencia de separación.

El emplear un valor para el exponente de la función de corrección de Richardson y Zaki del orden de 11 ( $n \leq$ 11), para partículas con un tamaño medio del orden de $1,0 \mu \mathrm{m}$, resulta adecuado ya que permite evaluar adecuadamente la velocidad de sumergimiento de conjuntos de micro partículas del tamaño de ese orden.

\section{REFERENCIAS BIBLIOGRÁFICAS}

1. DIN Norme 66111 Über Sedimentationsmethoden (1998): Norma DIN 66111 sobre Métodos de Sedimentación. Alemania.

2. Richardson J. F. and Zaki W. N. (1954). Sedimentation and fluidisation, Part I. Trans. Inst. Chem. Eng. 32, pp 35-53. Alemania.

3. Schubert H., Heidenreich E., Liepe F. (1990). Mechanische Verfahrenstechnik, Deutscher Verlag für Grundstoffindustrie, 2. Auflage. Alemania.

4. Zogg M. (1993). Einführung in die Mechanische Verfahrenstechnik, B. G. Teuner Stuttgart, 3. Auflage. Alemania. 